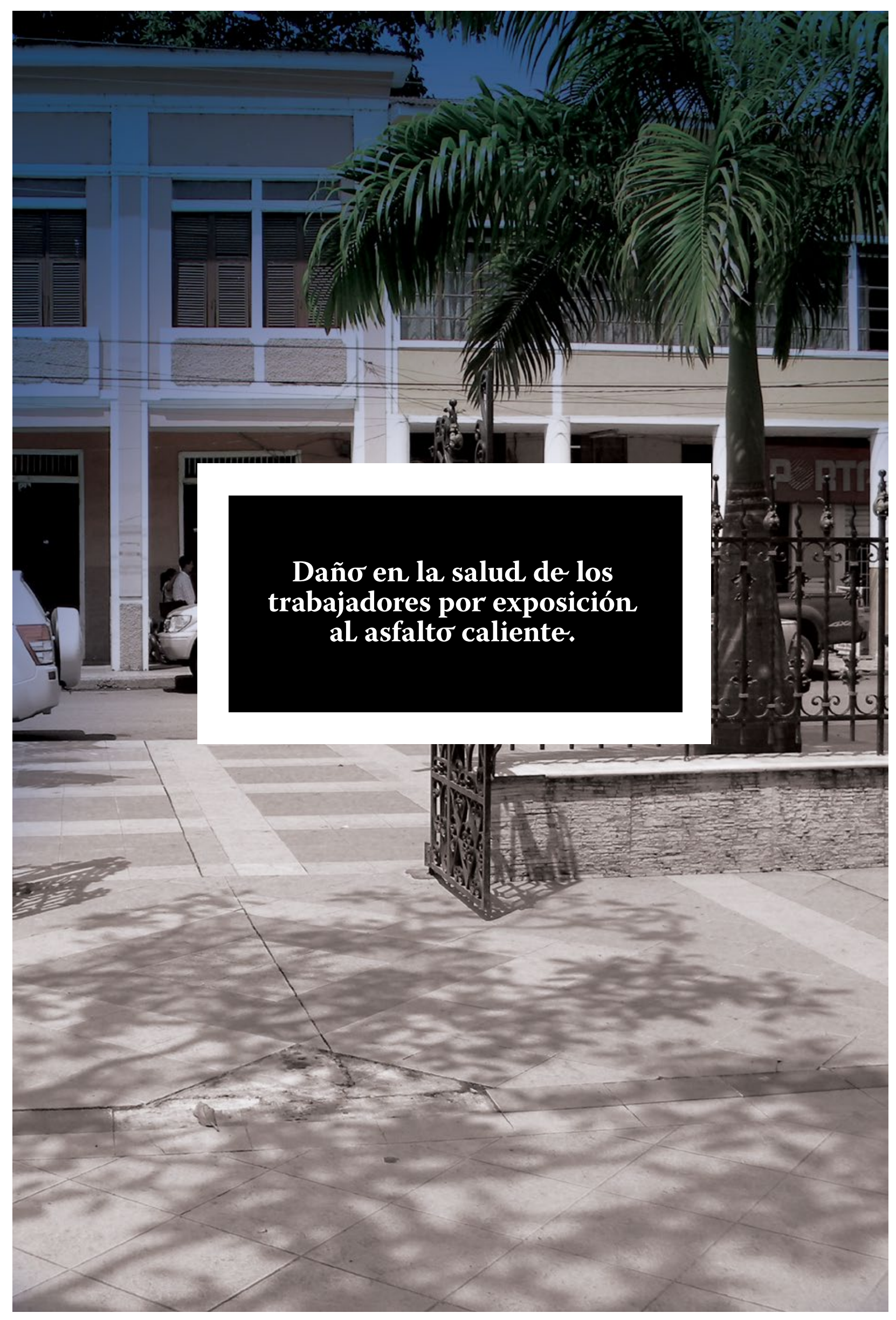




\title{
DAÑo EN LA SALUd DE LOS TRABAJAdorES POR EXPOSICIÓN AL ASFALTO CALIENTE
}

\author{
DAMAGE TO WORIERS 'HEALTH BY EXPOSURE TO HOT ASPHALT
}

\section{RESUMEN}

El presente trabajo intenta hacer una revisión sistemática sobre la bibliografía relacionada con la exposición laboral al asfalto caliente y el daño que ocasiona. El objetivo es determinar el impacto en la salud de los trabajadores por la exposición al calor del asfalto. Este propósito unido a la voluntad del hombre de manera ética y profesional conduce a información valiosa para la sociedad, estar al tanto de cifras conducentes que demuestran los efectos del asfalto a temperaturas elevadas, ocasiona la difusión del conocimiento como herramienta clave en la prevención y mitigación de efectos nocivos en la salud humana. Se direccionó la búsqueda en bases de datos bibliográficas como Latindex, Redib, Medline, Embase, Redalyc, Elsevier, Scielo; se consideró el título, la credibilidad y experiencia del autor, la idoneidad del resumen y la aplicabilidad de los resultados. A través de la intervención de distintas metodologías de análisis aplicadas en trabajadores de pavimentación, se demuestra la existencia de varios efectos como irritación en ojos, garganta, vías respiratorias y piel. El asfalto mezclado con alquitrán de hulla tiene mayor riesgo de exposición; el humo examinado en partículas atmosféricas demuestra asociación con disfunción anticipada del hígado, riñón y daños hematológicos, entre los hallazgos más representativos.

PALABRAS CLAVES: Asfalto caliente; daños en la salud; exposición laboral.

Copyright (c) Revista San Gregorio 2019. ISSN 1390-7247; eISSN: 2528-7907

\section{(c)}

\section{ABSTRACT}

The present work intends to make a systematic review about the literature related to occupational exposure to hot asphalt and the damage it causes. The objective is to determine the impact on workers' health due to exposure to asphalt heat. This purpose linked to the will of man in an ethical and professional manner leads to valuable information for society, to be aware of leading figures that demonstrate the effects of asphalt at high temperatures, causes the dissemination of knowledge as a key tool in the prevention and mitigation of harmful effects on human health. The search was addressed in bibliographic databases such as Latindex, Redib, Medline, Embase, Redalyc, Elsevier, Scielo; the title, credibility and experience of the author, the suitability of the summary and the applicability of the results were considered. Through the intervention of different analysis methodologies applied to paving workers, the existence of various effects such as irritation in eyes, throat, respiratory tract and skin is demonstrated. Asphalt mixed with coal tar has a higher risk of exposure; the smoke examined in atmospheric particles demonstrates association with anticipated liver, kidney and hemotological damage, among the most representative findings

KEYWORDS: hot asphalt; health damage; occupational exposure.

Copyright (c) Revista San Gregorio 2019. ISSN 1390-7247; eISSN: 2528-7907

1 Graduado de Maestría de Seguridad y Salud Ocupacional "Segunda

Cohorte" de la Universidad San Gregorio de Portoviejo. Manabí. Ecuador. 


\section{INTRODUCCIÓN}

El mayor beneficio del ecosistema versus, el adelanto y prosperidad del nivel de vida de las personas ha procurado un vasto conocimiento de los fenómenos existentes en el universo; de tal manera que el ser humano ha conseguido la mejora constante de cantidad, calidad y aplicación de nuevos productos y formas de transformación del saber científico. Sin embargo, parecería contradictorio que mientras tenemos mejores instrumentos para llevar una vida más placentera, por otro lado se activa una gran variedad de riesgos en la salud humana, sobre todo la manifestación de enfermedades derivadas del trabajo (Cortés, 2007).

Uno de los componentes descubierto por el ser humano es el asfalto, utilizado en el campo amplio de la ingeniería civil para ejecutar diversas actividades o construir obras de infraestructura. El asfalto que resulta del proceso de refinación del petróleo, ha sido un gran aliado en cuanto a labores viales se refiere, según el Manual del Asfalto (Instituto del Asfalto, 1973) esta sustancia conocida también como betún, es uno de los materiales más antiguos que el hombre ha empleado, tanto en pavimentación, revestimiento de obras hidráulicas y algunos otros usos.

El listado de enfermedades profesionales (Organización Internacional del Trabajo, 2010) contempla las causadas por los derivados alogenados de los hidrocarburos alifáticos o aromáticos, que justamente hace referencia al uso de asfalto; bajo esta premisa se hace necesario contemplar los riesgos que ocasiona en las personas que manipulan este elemento, en temas de salud y seguridad ocupacional es importante considerar el uso apropiado, la vestimenta, los utensilios adecuados; con el ánimo de minimizar el impacto del calor de una mezcla de asfalto al entrar en contacto con el ser humano.
La Organización Mundial de la Salud (OMS, 2017) menciona que 12,2 millones de personas en el mundo mueren en edad laboral por enfermedades no transmisibles, datos alarmantes que ponen de manifiesto el cuidado y la protección a considerar en los puestos de trabajo, y de manera muy especial el sector de la construcción.

Una parte central de la estimulación de daños y perjuicios a la salud del trabajador es el ambiente de trabajo, que según Cortés (2007) está compuesto por factores mecánicos, físicos, químicos y biológicos. El Reglamento de Seguridad y Salud para la Construcción y Obras Públicas (Ministerio de Trabajo y Empleo, 2008) establece todas las actividades de este sector, hace particular alusión a la vigilancia de la salud de los trabajadores. El texto especifica los cuidados y protección que se debe tener en cada una de las labores de la construcción, como el resguardo individual de cascos, ropa, mascarillas de acuerdo al tipo de exposición, y demás implementos.

El tema en cuestión aborda una problemática que para muchos es desconocida, sin embargo, poco a poco ha despertado interés por la gran cantidad de enfermedades generadas a nivel mundial. La investigación por su parte ayuda a tomar medidas concretas a través del conocimiento que se desprende de diversas pruebas e instrumentos empleados, los resultados ayudan a la humanidad a descubrir efectos en la salud que día a día aparecen y por lo consiguiente la información sirve para tomar medidas de precaución y hacer conciencia al momento de exponerse al contacto del calor de asfalto.

El presente trabajo busca determinar el impacto en la salud de los trabajadores por la exposición al calor del asfalto; a través de una revisión sistemática, síntesis e interpretación del contenido.

\section{METODOLOGÍA}

La investigación aplicada a este trabajo científico es de revisión sistémica a través de la cual se busca información bibliográfica sobre los efectos que provoca la exposición al calor del asfalto en quienes trabajan directamente en esta labor. La búsqueda fue direccionada a encontrar información en base de datos bibliográficas como Latindex, Redib, Medline, Embase, Redalyc, Elsevier, Scielo. Además, se obtuvo datos en documentos, enciclopedias y 
trabajos investigativos del Instituto Tecnológico de Costa Rica, Universidad de Carabobo, Universidad de Oviedo, Universidad Autónoma Agraria Antonio Navarro, Asociación para el Avance de la Ciencia y la Tecnología en España, Universidad del Azuay, Universidad Fasta y la Universidad Nacional Abierta y a Distancia, con fechas de publicación no menor al año 2005, en idioma español e inglés. Las fechas de consulta fueron entre octubre y diciembre de 2018, inicialmente se identificaron 50 trabajos científicos que trataban la temática abordada, con los que se realizó una lectura rápida. Más adelante al efectuar una lectura crítica y de mayor análisis, se consideró que 30 artículos y tesis referían a investigaciones centradas específicamente en los daños que provoca el humo de varios hidrocarburos aromáticos policíclicos (PAHs) al entrar en contacto con las personas, y finalmente, a través del criterio del autor se consideró que 20 de ellos corresponden a los relacionados con efectos en la salud humana por la exposición a asfalto caliente.

Se empleó descriptores como riesgo, asfalto caliente, daño en la salud. Para el caso de artículos científicos se procedió a observar el título, la credibilidad y experiencia del autor, la idoneidad del resumen y la aplicabilidad de los resultados.

Los criterios de inclusión: estudios en personas con diez años mínimo dedicados a la colocación de asfalto, medidas de seguridad y protección aplicadas, recomendaciones técnicas por parte de entidades involucradas, estudios publicados en inglés y español.

Los criterios de exclusión: estudios en trabajadores de pavimentos distintos al asfalto, artículos que no tratan sobre exposición ocupacional.

Los criterios confusoras: antecedentes cancerígenos en familiares, estilos de vida y alimentación no saludable, investigaciones con datos tomados en personas fumadoras.

\section{RESULTADOS}

El asfalto según (Mastandrea, y otros, 2005) es considerado un hidrocarburo aromático policíclicos (PAHs) producto de diversos procesos industriales, sus compuestos son cancerígenos y/o mutágenos, es importante el control a su exposición. Este componente es elaborado principalmente a base de petróleo, que una vez mezclado con otros agentes se calientan a temperaturas muy elevadas, absorbidas por quienes ejecutan esta labor.

La enciclopedia de salud y seguridad en el trabajo condensa la información de localización de tipos de cánceres de acuerdo a la industria en la que se trabaje, en el caso de trabajadores de asfalto que está dentro de la industria 5, Construcción, menciona el tipo de cáncer más común, que es el de pulmón, cuyo agente causal son los hidrocarburos aromáticos policíclicos (PAHs) (Pearce, Boffetta \& Kogevinas, s.f). Para la Organización Mundial de la Salud el cáncer es la razón más repetitiva de muertes en el mundo, según datos de este organismo en el año 2015 existe un aproximado de 8,8 millones de personas que murieron a causa de esta enfermedad, y específicamente el cáncer de pulmón, representó 1,69 millones de muertes (Organización Mundial de la Salud, 2018). Este organismo así mismo hace mención a los diferentes riesgos que hacen más probable la generación de cáncer, y entre otros recalca la ingesta de alimentos no saludables, la inactividad física o vida sedentaria, la utilización de cigarrillo y alcohol.

Los daños en las vías respiratorias, la garganta y los ojos, ocasionados por el humo de asfalto en trabajadores, han sido temas de interés para varios investigadores, es así que resultados de varios estudios lo asocian con irritación ocular y efectos en el aparato respiratorio. Para (Cuesta, Martínez \& Espinosa, 2016) aplicó cuestionarios de síntomas respiratorios, y auto reportes de síntomas oculares a 135 trabajadores de una empresa de infraestructura vial, concluyeron que se debe alertar las propiedades toxicológicas y de manera directa el benzo(a)pireno en la actividad de colocación de asfalto. De la misma manera (Moscote, Méndez \& Beltrán, 2012) evaluaron a 985 trabajadores a través de la Guía Técnica Colombiana, encontraron que el humo del asfalto caliente provoca efectos irritantes en los ojos desde el panorama toxicológico industrial, además de secreción nasal y ronquera (Rumler, Rühl, Nies, Rode \& Heger, 2007) dentro de su investigación daños en el aparato respiratorio y ojos por la exhibición laboral de asfalto, recomiendan el uso de mecanismos de protección ante tal efecto (Medina \& Rodríguez, 2009). 
A partir de un análisis de espirometría líquido de lavado nasal y esputo inducido, efectuado a 320 trabajadores expuesto al betún caliente (Raulf-Heimsoth y otros, 2011) pretenden descubrir los efectos que esta labor puede ocasionar en los pulmones. Para (Olsson y otros, 2010) los resultados se ajustan a tres parámetros examinados, como son la función pulmonar, la capacidad vital forzada (FVC) y el volumen espiratorio forzado en un segundo (FEV1) los valores dentro de los rangos normales, siendo las principales deducciones del estudio:

a.- Ninguna asociación significativa entre los indicadores de inhalación y exposición dérmica al betún y el cáncer de pulmón.

b.- La falta de un efecto de otros carcinógenos pulmonares ocupacionales conocidos o presuntos en la industria del asfalto o en otros trabajos, con la posible excepción de la exposición al alquitrán de hulla.

c.- Mayor prevalencia de tabaquismo en la población del estudio en comparación con las encuestas nacionales, lo que podría haber sesgado los resultados del estudio de cohorte lejos de la nula.

Las interrogantes sobre los posibles riesgos cancerígenos producidos por el humo de asfalto en trabajadores que se exponen a este producto permitió que el Instituto Nacional de Seguridad y Salud Ocupacional apoyara dos bioensayos en la piel de ratones, los estudios mostraron que todos los condensados de humo y dos de las cinco subfracciones de HPLC del humo de temperatura más alta eran carcinogénicos. A partir de ello (Kriech, Kurek \& Wissel, 2009) diseñaron una investigación para establecer hasta qué punto el modo de generación de humo de NIOSH afectó su composición y, por lo tanto, la actividad biológica. Los resultados mostraron que pequeños cambios en el modo de generación producen marcadas diferencias en la composición del humo. Los humos generados en el laboratorio se enriquecieron significativamente en PAC de mayor peso molecular asociados con carcinogenicidad en estudios con animales.

Otro estudio de (Rodríguez, 2011) permitió evaluar la generación de cáncer de pulmón laboral, la autora recopiló el análisis de 854 casos y 809 controles, de los cuales dentro de las tareas y los procesos de trabajo respecto a la exposición de (PAHs), por asfalto se presentaron 63 casos y 53 controles. Los resultados determinaron que la falta de reconocimiento del cáncer laboral de pulmón no permite tomar medidas para la prevención efectiva de esta enfermedad ocupacional.

En las investigaciones efectuadas en el Ecuador, se destaca la tesis de Rodas (2016) que consistió en evaluar a 15 trabajadores directos del revestimiento de asfalto en la obra Vía Colimes - Olmedo durante julio 2013 hasta julio 2015. Dicha investigación concluyó que los accidentes y enfermedades laborales en trabajadores de asfalto son sobre todo por: exposición a gases por la mezcla de asfalto, manipulación de mezcla asfáltica caliente y combustión por error de manipulación y ries gos físicos.

A través de una regresión logística incondicional (Karami y otros, 2011) midieron el riesgo en relación con la exposición laboral a ciertos hidrocarburos aromáticos policíclicos y su incidencia en la determinación de cáncer renal (carninomas de células renales), concluyeron que hay una posible asociación entre el estireno ocupacional y la exposición al acrilonitrilo y el riesgo de cáncer renal. Sin embargo, se necesitan estudios adicionales para replicar los hallazgos, ya que es la primera vez que se informan estas asociaciones y pueden deberse al azar.

\section{DISCUSIÓN}

La exposición al asfalto caliente provoca enfermedades en la piel, en los ojos (Cuesta, Martínez \& Espinosa, 2016) y según varias investigaciones este elemento repercute en otros órganos como garganta, pulmones e incluso en el ADN de las personas. Los estudios realizados y los métodos empleados hasta la actualidad han permitido corroborar la hipótesis sobre si el humo de asfalto perjudica la salud del hombre, sobre todo de aquellos que se exponen laboralmente a este PAHs. Aquellos trabajos científicos que han utilizado la recaudación de datos a través de cuestionarios, observaciones, bioensayos, muestras de sangre, orina y manifestación en el ambiente; nos traen resultados que alertan los posibles riesgos a los que se enfrentan día a día los trabajadores en la pavimentación. Cuesta, Martínez \& Espinosa (2016) consideran que la protección respiratoria, la ropa de trabajo y los guantes presentan mayor aceptabilidad al 
uso, sin embargo esto puede ser tomado simplemente como reglas que la empresa deben cumplir, más no como medidas de protección de la salud humana. Conscientes que los trabajadores que manipulan asfaltos, realizan además limpieza de la calzada o la retirada de pavimentos deteriorados, por lo que pueden estar sometidos a la inhalación del polvo siendo conveniente el control de este peligro.

En la revisión efectuada se denotan gran interés por el uso de métodos de intervención a través de muestras de sangre y orina en su gran mayoría, además de congregaciones atmosféricas y aplicación de encuestas sobre síntomas y reportes de enfermedades; dentro de la investigación existen resultados que demuestran la poca o nula certeza de efectos cancerígenos, sobre todo de pulmón a causa del asfalto (Olsson y otros, 2010) entre los que podemos destacar:

1.- La PAC (compuestos aromáticos policíclicos) mediana en fase de vapor $(5,5 \mu \mathrm{g} \mathrm{m}$ - 3), PAHs ( $\leq 50$ ng m - 3) y OH- PAHs $(0,08-$ $1,11 \mu g 1-1)$ significativamente más altas en $\mathrm{AW}$ que en CW. Los niveles de $\mathrm{OH}$ - PAHs durante el día laboral y la semana laboral 0.29, 0.08 y 0.18 al inicio del estudio; 0.50, 0.18 y 0.29 , turno previo; $1.11,0.44$ y $0.44 \mu \mathrm{g} 1-1$, post-turno (Buratti y otros, 2007).

2.- Fallecidos de cáncer de pulmón entre los años 1980 hasta 2005, con una exposición de humo y el condensado bituminoso, el vapor orgánico y los hidrocarburos aromáticos policíclicos, así como para el asbesto, la sílice cristalina, los gases de escape de motores diésel y el alquitrán de hulla, muestra resultados de 1.12 [intervalo de confianza (IC) del 95\%, 0.84-1.49] para la exposición por inhalación al humo de betún y 1.17 [intervalo de confianza (IC) del 95\%, 0.88-1.56] para la exposición dérmica al condensado de betún.

No obstante, debemos estar alertos siempre a los riesgos que implica exponerse a productos que se derivan del petróleo; pero para el caso de los PAHs se precisa que existe relación directa entre tiempo de exposición y riesgo de este tipo de cáncer (Rodríguez, 2011). En algunos casos también existen rangos de nivel moderado o normales en la función pulmonar, capacidad vital forzada y volumen respiratorio (Raulf-Heimsoth y otros, 2011). Como factores limitantes dentro de la revisión se pudo constatar la falta de va- riedad de artículos que traten la temática a partir del año 2005, existen muy pocos estudios efectuados en nuestro país y en toda Latinoamérica, más bien en su gran mayoría son de autores norteamericanos y europeos, entre otros. Esta situación podría sesgar los valores y deducciones por existir diferencias por ejemplo en los hábitos alimenticios, mayor concentración de contaminantes en el ambiente, entre otros factores.

Los aportes de las investigaciones efectuadas respecto al tema en cuestión, deben ser confrontadas con los datos estadísticos que presentan organismos internacionales relacionados con temas de salud ocupacional, éstos actualmente son alarmantes sobre todo en el sector de la construcción, he aquí un problema muy complejo porque cada nación presenta panoramas distintos en su medio ambiente, leyes, cultura, nivel de formación y el enfoque que se le da a un criterio tan importante, como es el daño que ocasiona el trabajo con humo de asfalto. Por un lado, es beneficioso tener carreteras de primer orden, pero por otro lado hay que, tomar las medidas respectivas que minimicen, o de ser el caso eliminen todo riesgo de contraer enfermedades en el corto, mediano y largo plazo en este importante sector de la economía de un país.

Existen resultados investigativos y cifras o padrones, pero lo que ciertamente aún no se tiene es una cultura de prevención y toma de conciencia de los efectos que puede ocasionar la exposición a temperaturas elevadas mientras se coloca asfalto en las vías o carreteras; en la mayoría de los casos por desconocimiento a profundidad sobre los mismos; se considera un trabajo sin mayores complicaciones en términos de salud, pero que en ocasiones puede provocar con el paso del tiempo el desencadenamiento de enfermedades crónicas.

\section{CONCLUSIONES}

La tecnología y sus diferentes adelantos sirven para la realización de las actividades del ser humano de manera más eficiente y productiva en todos los campos de la ciencia; sin embargo, muchos de los elementos que se integran en las labores diarias provienen de la mezcla de sustancias químicas y compuestos biológicos que de alguna $u$ otra manera repercute en el bienestar de las personas. Con la revisión efectuada y los hallazgos encon- 
trados por los diferentes autores se considera que:

Los efectos que ocasiona el humo de asfalto en el ser humano son variados, debido a su contenido de petróleo se vuelve un material de trabajo tóxico, por tanto los factores por efecto de antecedentes familiares con cáncer, si bien es cierto podrían tener una relación con la generación de esta enfermedad, pero el aspecto más importante es el alto grado de agentes cancerígenos que poseen todos los derivados de petróleo.

Es importante y necesario efectuar un seguimiento constante en los trabajadores que manipulen asfalto caliente, monitorear su salud, resguardar su integridad física e informar sobre los efectos y las precauciones que deben tener presente al momento de realizar sus tareas con esta sustancia. Así mismo, los hábitos de alimentación de las personas juegan un papel preponderante en la prevención o generación de diversas enfermedades, y mucho más específico el cáncer, entonces es importante hacer conciencia en los trabajadores de asfalto, que, si unimos esto con el efecto que tienen los agentes químicos del asfalto, se vuelve un efecto con mayor riesgo.

El consumo de alcohol y cigarrillos ejerce también una fuerte relación, se podría decir la más importante al momento de diagnosticar enfermedades no transmisibles, ocupacionales y mortales; por ello los trabajadores que mantienen un contacto directo con humo de asfalto presentan mayores probabilidades de enfermar y mucho más latente aquellas que resultan ser mortales.

\section{REFERENCIAS BIBLIOGRÁFICAS}

Agostini , M., Ferro , G., Burstyn , I., de Vocht, F., Portengen , L., Olsson , A., .. . Kromhout, H. (2013). Does a more refined assessment of exposure to bituminous fumes and confounding factors alter the risk estimates of a nested case-control study of lung cancer among European asphalt workers? Occup Environ Med., 195202

Bacaksiz, A., Kayaalti, Z., Soylemez, E., Tutkun, E., \& Soylemezoglu, T. (2014). Lymphocyte DNA damage in Turkish asphalt workers detected by the comet assay. International Journal of Environmental Health Research, 11-17.

Buratti, M., Campo, L., Fustinoni, S., Cirla, P., Martinotti, I., Cavallo, D., \& Foa, V. (2007). Urinary hydroxylated metabolites of polycyclic aromatic hydrocarbons as biomarkers of exposure in asphalt workers. Biomarkers, 221-239.

Campo, L., Addario, L., Buratti, M., Scibetta, L., Longhi, O., Valla, C., . . . Fustinoni, S. (2006). Biological monitoring of exposure to polycyclic aromatic hydrocarbons by determination of unmetabolized compounds in urine. Toxicology Letters, 132-138.

Cavallari, J., Osborn, L., Snawder, J., Kriech, A., Olsen, L. Herrick, R., \& Mcclean, M. (2012). Predictors of Airborne Exposures to Polycyclic Aromatic Compounds and Total Organic Matter among Hot-Mix Asphalt Paving Workers and Influence of Work Conditions and Practices. The Annals of Occupational Hygiene, 138-147.

CAVALLO, D., URSINI, C. L., BAVAZZANO, P., CASSINELLI, C., FRATTINI, A., PERNICONI, ,. B., . . . IAVICOLI, S. (2006). Sister Chromatid Exchange and Oxidative DNA Damage in Paving Workers exposed to PAHs. The Annals of Occupational Hygiene, 211-218.

Chong, D., Wang, Y., Guo, H., \& Lu, Y. (26 de noviembre de 2014). Volatile Organic Compounds Generated in Asphalt Pavement Construction and Their Health Effects on Workers. Recuperado el 25 de noviembre de 2018, de https://ascelibrary.org/doi/

abs/10.1061/\%28ASCE\%29CO.1943-7862.0000801
Cirillo, T., Arnese, A., Del Prete, E., Del Prete, U., \& Amodio, R. (2007). Evaluation of airborne respirable particulate matter and polycyclic aromatic hydrocarbon exposure of asphalt workers. I T A L I A N J O U R N A L O F P U B L I C H E A L T H, 278-284.

Cirla, P., Martinotti, I., Buratti, M., Fustinoni, S., Campo, L., Zito, E., ... Foà, V. (2007). Assessment of Exposure to Polycyclic Aromatic Hydrocarbons (PAH) in Italian Asphalt Workers. Journal of Occupational and Environmental Hygiene, 87-99.

Cortés, J. (2007). Técnicas de Prevención de Riesgos Laborales Seguridad e Higiene del Trabajo. Madrid: Tebar.

Cuesta, J., Martínez, L., \& Espinosa, M. (2016). EFECTOS IRRITANTES EN PIEL, MUCOSAS RESPIRATORIAS Y OCULARES EN TRABAJADORES EXPUESTOS A HUMOS DE ASFALTO DE UNA EMPRESA DE INFRAESTRUCTURA VIAL - CUNDINAMARCA, COLOMBIA. Salud Bosque, 7-22.

Instituto del Asfalto. (1973). Manual del Asfalto. Bilbao, España: Urmo S.A. Recuperado el 20 de noviembre de 2018, de Manual del Asfalto: https://es.scribd.com/ doc/123896961/Manual-Del-Asfalto

Karaman, A., \& Pirim, I. (2009). Exposure to bitumen fumes and genotoxic effects on Turkish asphalt workers. Clinical Toxicology, 321-326.

Karami, S., Boffetta, P., Brennan, P., Stewart, P., Zaridze, D., Matveev, V., ... Moore, L. (2011). Renal cancer risk and occupational exposure to polycyclic aromatic hydrocarbons and plastics. Author Manuscript, 218-223.

Kriech, A., Kurek, J., \& Wissel, H. (2009). Effects of Mode of Generation on the Composition of Asphalt Fumes. Polycyclic Aromatic Compounds, 179-188.

Marczynski, B., Raulf-Heimsoth, M., Pesch, B., Kendzia, B., Käfferlein, H., Vosshans, B., .. . Brüning, T. (2010). Detection of DNA strand breaks by comet assay in sputum leucocytes of bitumen-exposed workers: A pilot study. Human \& Experimental Toxicology, 721-729. 


\section{REFERENCIAS BIBLIOGRÁFICAS (CONT.)}

Mastandrea, C., Chichizola, C., Ludueña, B., Sánchez, H., Álvarez, H., \& Gutierrez, A. (1 de Enero de 2005). Hidrocarburos aromáticos policíclicos. Riesgos para la salud y marcadores biológicos. 27-36. Buenos Aires, Argentina. Recuperado el 12 de diciembre de 2018, de https://www.redalyc.org

MCCLEAN, M., WIENCKE , J., KELSEY, K., VARKONYI, A., ONG, L., EISEN, E., \& HERRICK, R. (2006). DNA Adducts among Asphalt Paving Workers. The Annals of Occupational Hygiene, 27-34.

Medina, M., \& Rodríguez, M. (2009). Determinación de la exposición a humos de asfalto en trabajadores de carreteras. Guanacaste, Heredia, Cartago y San José Instituto Tecnológico de Costa Rica.

Ministerio de Trabajo y Empleo. (10 de Enero de 2008). Registro Oficial N ${ }^{\circ} 249$. Reglamento de Seguridad y Salud para la Construcción y obras Públicas. Quito, Pichincha, Ecuador. Recuperado el 15 de diciembre de 2018, de http://www.trabajo.gob.ec

Moscote, O., Méndez, G., \& Beltrán, C. (2012). ANÁLISIS DE ACCIDENTES DE TRABAJO Y ENFERMEDADES PROFESIONALES EN ACTIVIDADES DE PAVIMENTACIÓN CON ASFALTO. Redes de Ingeniería, 66-81.

Neghab, M., Derisi, F., Forough, Z., Hassanzadeh, J. Dirin, V., \& Heidari, S. (2016). Toxic responses of different organs following occupational exposure to subthreshold limit value levels of paving asphalt fumes. TOXICOLOGICAL \& ENVIRONMENTALCHEMISTRY 331-339.

Olsson, A., Kromhout, H., Agostini, M., Hansen, J., Funch , C., Johansen, C., . . . Boffetta, P. (2010). A Case-Control Study of Lung Cancer Nested in a Cohort of European Asphalt Workers. Environ Health Perspect, 1418-1424.

OMS. (30 de Noviembre de 2017). Protección de la salud de los trabajadores. Recuperado el 23 de noviembre de 2018, de http://www.who.int/es/news-room/fact-sheets/ detail/protecting-workers'-health

Organización Internacional del Trabajo. (2010). Lista de Enfermedades Profesionales de la OIT. Conferencia General de la OIT (pág. 4). Ginebra: Organización Internacional del Trabajo. Recuperado el 14 de diciembre de 2018, de https://www.ilo.org/wcmsp5/groups/ public/@ed_protect/@protrav/@safework/documents/ publication/wcms_125164.pdf

Organización Mundial de la Salud. (12 de Septiembre de 2018). Cáncer. Recuperado el 27 de enero de 2018, de https://www.who.int/es/news-room/fact-sheets/detail/ cancer
Pearce, N., Boffetta, P., \& Kogevinas, M. (S/A). El cuerpo humano. En Enciclopedia de salud y seguridad en el trabajo (págs. 1-19). Madrid: Instituto de Segurdidad y Salud Ocupacional.

Raulf-Heimsoth, M., Pesch, B., Kendzia, B., Spickenheuer, A., Bramer, R., Marczynski, B.,Brüning, T. (2011). Irritative effects of vapours and aerosols of bitumen on the airways assessed by non-invasive methods. Archives of Toxicology, 41-52.

Rodas, G. (2016). DETERMINAR LOS FACTORES DE RIESGO FISICOS A LOS QUE ESTAN EXPUESTOS LOS TRABAJADORES RESPONSABLES DEL REVESTIMIENTO ASFALTICO Y PROPUESTA DE SEGURIDAD Y SALUD OCUPACIONAL DIRIGIDO A LOS TRABAJADORES DE LA VIA EN LA COMPAÑIA VERDU S.A. Guayaquil: Universidad de Guayaquil.

Rodríguez, F. (2015). RIESGOS EMERGENTES EN LA INDUSTRIA DE LA CONSTRUCCION EN TRABAJADORES MAYORES A 50 AÑOS. Carabobo: Universidad de Carabobo.

Rodríguez, M. (2011). Cáncer de Pulmón por Hidrocarburos Aromáticos Policíclicos en la población del estudio Capua. Oviedo: Universidad de Oviedo.

Rumler, R., Rühl, R., Nies, E., Rode, P., \& Heger, M. (2007). Health Complaints of German Mastic Asphalt Workers. Journal of Occupational and Environmental Hygiene, 233-236.

Serdar, B., Lee, D., \& Dou, Z. (2012). Biomarkers of exposure to polycyclic aromatic hydrocarbons (PAHs) and DNA damage: a cross-sectional pilot study among roofers in South Florida. BMJ, 1-10.

Sobus, J., Mcclean, M., Herrick, R., Waidyanatha, S., Onyemauwa, F., Kupper, L., \& Rappaport, S. (2009). Investigation of PAH Biomarkers in the Urine of Workers Exposed to Hot Asphalt. The Annals of Occupational Hygiene, 551-560.

Sudha, S., Bhuvaneswari, M., \& Kripa, K. (2011). Cytogenetic Biomonitoring of Road Paving Workers. Asian Pacific Journal of Cancer Prevention, 713-717.

Trumbore, D., Osborn, L., Johnson, K., \& Fayerweather, W. (2015). Airborne Exposures to Polycyclic Aromatic Compounds Among Workers in Asphalt Roofing Manufacturing Facilities. Journal of Occupational and Environmental Hygiene, 564-576.

Väänänen, V., Hämeilä, M., Kalliokoski, P., Nykyri, E., \& Heikkilä, P. (2005). Dermal Exposure to Polycyclic Aromatic Hydrocarbons among Road Pavers. The Annals of Occupational Hygiene, 167-178. 